\title{
Optical event horizon based complete transformation and control of dark solitons
}

\author{
Zhixiang Deng, ${ }^{1,2}$ Yu Chen, ${ }^{1}$ Jun Liu, $,{ }^{1,3},{ }^{*}$ Chujun Zhao, ${ }^{4}$ and Dianyuan Fan ${ }^{1}$ \\ ${ }^{1}$ International Collaborative Laboratory of 2D Materials for Optoelectronics Science and Technology, Key Laboratory of Optoelectronic Devices \\ and Systems of Ministry of Education and Guangdong Province, College of Optoelectronic Engineering, Shenzhen University, Shenzhen 518060, \\ China \\ ${ }^{2}$ School of Electrical Engineering, University of South China, Hengyang 421001, China \\ ${ }^{3}$ Aston Institute of Photonic Technologies, School of Engineering and Applied Science, Aston University, Birmingham B4 7ET, U.K. \\ ${ }^{4}$ Laboratory for Micro-/Nano- Optoelectronic Devices of Ministry of Education, IFSA Collaborative Innovation Center, School of Physics and \\ Electronics, Hunan University, Changsha 410082, China \\ *Corresponding author: j.liu26@aston.ac.uk
}

Received XX Month XXXX; revised XX Month, XXXX; accepted XX Month XXXX; posted XX Month XXXX (Doc. ID XXXXX); published XX Month XXXX

We propose a manipulation approach to vary the wave speed as well as the grayness of dark solitons under the optical event horizon arising from the interaction between a dark soliton and a probe wave. The optical event horizon effect is demonstrated, for the first time to be capable of inducing a reversible conversion between a black soliton and a gray one. This reversible soliton transformation and control process originates from the intrinsic competition between the probe-induced nonlinear phase shift and the internal phase of the dark soliton. In a cascaded system consisting of two optical event horizons, we also observe the new optical soliton tunneling phenomena where a dark soliton can be reset longitudinally purposely. The results may find applications in information cloaking such as effectively hiding the presence of intermediate fiber section to the receiver. (C) 2018 Optical Society of America

OCIS codes: (190.4380) Nonlinear optics; (060.4370) Nonlinear optics, fibers; (190.5530) Pulse propagation and temporal solitons.

http://dx.doi.org/10.1364/OL.99.099999

Optical event horizons based on the nonlinear interaction between solitons and weak probe waves in optical fibers is an active field of research [1-4] and open innovative perspectives in light control [58]. The typical optical event horizons are formed when a weak probe wave, traveling at a different group velocity with respect to an intense soliton, is unable to pass through it during their collision. This intrinsic mechanism was described in terms of the solitoninduced refractive index barrier (Kerr effect) [1] that leads to the temporal reflection of the probe wave onto the edge of the energetic soliton, and thus alters the speed of the probe pulse. The speed change of the probe wave is directly resulting from the occurrence of a reversible frequency conversion between the weak probe wave and the reflected wave in the regime of the optical event horizon [2, 9]. The key point of the optical event horizon lies in the temporal reflection process, which does not only affect the frequency and group-velocity of the weak probe wave, but also leads to the propagation manipulation of the energetic soliton pulse [1]. Researchers found that the duration, intensity, and carrier frequency of a soliton can be modified by adjusting the probe wave through certain parameters [5, 10-13]. Based on this the optical event horizon can be used to control the formation of the fiberoptical rogue waves [14]. Interestingly, it has been numerically [15, 16] and experimentally [17] shown that the nonlinear mechanism can generate a solitonic cavity formed by two solitons to trap a weak probe wave. Recently, we also numerically and analytically demonstrated that it is possible to achieve a dark soliton transformation (from dark soliton to gray one) through the temporal reflection process in Ref. [18] where the linear-stability of dark solitons under the probe wave perturbation was carefully analyzed.

Dark solitons are one-dimensional non-dispersive "negative pulses" formed by the balance between the dispersion in the normal-dispersion range and the Kerr nonlinearity, exhibiting a dip in a nonzero uniform background [19]. The experimental demonstration of data transmission based on dark solitons [20] has led to renewed efforts to explore this type of soliton for optical communications. Although the generation of a modulated stream of dark solitons is a far more difficult task in contrast to the bright ones, dark solitons are usually believed to have more advantages in terms of loss, noise, and mutual interactions between adjacent pulses [2123]. In earlier studies it was shown that synchronous phase modulation [22] and nonlinear gain [23] are effective in controlling the time shift of dark solitons caused by incomplete grayness of the solitons. The dark soliton transmission control is still attracting researcher' increased interest.

In this letter, we demonstrate a method to completely manipulate the probe-controlled dark soliton transformation based 
on optical event horizon. The reversible conversion between a black soliton and a gray one with different grayness can be achieved through adjusting the weak probe wave. We show that the dark soliton can be reset at will using optical event horizon and the input black/gray soliton recovered.

Let us begin our study with numerical simulations based on the following normalized nonlinear Schrödinger equation (GNLSE) [21]:

$$
\partial_{Z} A+\frac{i}{2} \partial_{T}^{2} A+\delta_{3} \partial_{T}^{3} A=i|A|^{2} A,
$$

where $T=\left(t-z / v_{g}\right) / T_{0}$ is the normalized retarded time for the soliton traveling at group velocity $v_{g}$, and $Z=z / L_{D}$ is the normalized distance with dispersion length $L_{D}=T_{0}^{2} / \beta_{2}$. The dimensionless coefficient $\delta_{3}=\beta_{3} /\left(6 T_{0} \beta_{2}\right)$ stands for the relative strength of the third-order dispersion, which is required to obtain the regime of optical event horizon. We neglected higher-order dispersion terms ( $\left.\beta_{n}, \mathrm{n}>4\right)$, self-steepening and Raman effects since they do not play any significant role in the manipulation technique involved in this work. Although the Raman effect clearly affects the dark soliton propagation, such as decreasing its amplitude, it is neither an inevitable necessity for control and transformation of dark solitons, nor of much importance for the main concept of an optical event horizon with light-by-light control [24].

If we consider $\delta_{3}=0$, Eq. (1) is integrable and admits the following dark soliton solution $A_{d s}(Z, T)$ of the form [17]

$$
\begin{gathered}
A_{d s}(Z, T)=u_{0} \cdot(\cos \phi \tanh \Theta-\dot{\sin } \phi) e^{\dot{u_{0}^{2} Z},} \\
\Theta \equiv u_{0} \cdot\left(T-Z \cdot u_{0} \sin \phi\right) \cos \phi,
\end{gathered}
$$

where $u_{0}=1$ is the background wave amplitude, and $\phi(|\phi| \leq \pi / 2)$ is the internal phase angle that is related to the grayness parameter of the dark soliton $(\cos \phi)$ as well as the velocity $(\sin \phi)$. Black soliton are termed if $\phi=0$ and gray solitons otherwise. Dark solitons are also associated with a phase jump localized at the dip center. A black soliton has an abrupt $\pi$ phase jump while the phase change becomes smoother and smaller for gray solitons. Furthermore, it is noteworthy that when $\phi<0$ the soliton travels faster than the carrier frequency, otherwise the soliton slower. In the presence of third-order dispersion, it is generally known that the dark soliton emits resonant radiation [2529] similarly to bright solitons when the phase matching condition is satisfied. However, we have chosen $\delta_{3}=0.029$ here, the energy emission may be so small that the impact of third-order dispersion on a dark soliton is disregarded [25].

To study the possibility of manipulating dark solitons by a weak probe wave, we consider an input consisting of two launched pulses with different frequencies, namely the dark soliton and the weak probe wave, as following:

$$
A(0, T)=A_{d s}(0, T)+A_{p}(0, T) .
$$

The probe wave is given by

$$
A_{p}(0, T)=\sqrt{P_{p}} \exp \left(-\left(T-T_{1}\right)^{2} / w^{2}\right) \cdot \exp \left(-i \omega_{p} T\right),
$$

where $P_{p}$ is the normalized peak power of the probe wave, $T_{1}$ is the temporal delay relative to the dark soliton, $w=10$ is the temporal width of probe wave, and $\omega_{p}=-10.4$ is the relative frequency of incident probe wave. A typical simulated temporal dynamics of the interaction between a black soliton and a weak probe wave is presented in Fig. 1(a) and 1(c). As can be seen from Fig. 1(c), at around the collision point $(\mathrm{z} \approx 38$ ), the probe wave is partially reflected on the edge of the black soliton, after which the soliton is accelerated relative to its original trajectory. Figure 1(a) shows the intensity profiles of the input and output dark soliton before and after the temporal reflection process occurs, and indicates that the quasi-stationary black soliton ( $\sin \phi=0)$ is transformed into a gray soliton ( $\sin \phi=-0.2$ ), which travels faster than the carrier frequency. This transformation process is significantly different from the evolution mechanism for even dark pulse that will eventually split into a pair of gray solitons [30].
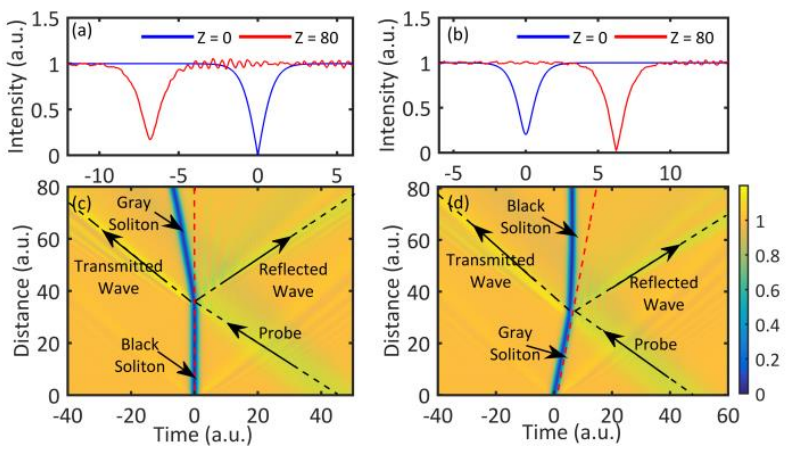

Fig. 1. Numerical simulation of the collision dynamics between a dark soliton and a weak probe wave. (a, c) correspond to the case of a black soliton interacting with a weak probe wave $\left(\mathrm{P}_{\mathrm{p}}=0.016\right)$, showing that the black soliton is transformed into a gray one ( $\sin \phi=-0.2)$ under the action of the weak probe wave. $(b, d)$ represent the interaction between a gray soliton and a weak probe wave $\left(\mathrm{P}_{\mathrm{p}}=0.026\right)$, showing that a gray soliton can be transformed into a black one. The dashed red lines in (c, d) indicate the trajectories without the action of probe waves.
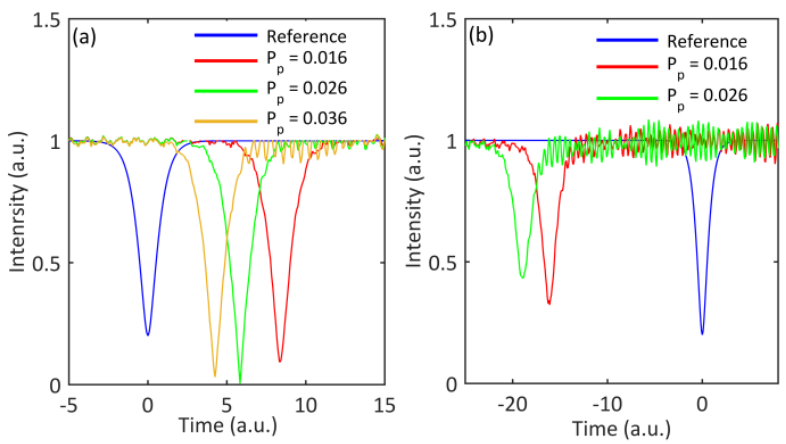

Fig. 2. Output temporal profiles of the dark soliton manipulated by the probe wave with different peak powers. (a) and (b) correspond to the situations where a slower and faster dark soliton relative to the velocity at the carrier frequency interact with the probe wave, respectively.

The natural transformation of a black soliton into a gray one makes us to further investigate the possibility to achieve its inverse process from the gray soliton to a black one under the action of a probe wave. In this sense, we apply the converted gray soliton ( $\sin \phi=0.2$ ) as the input dark soliton. Figure 1(b) and 1 (d) show the dynamics of the interaction between the gray soliton and the 
weak probe wave. It is clearly seen in Fig. 1(d) that the reflection of the probe wave pushes the gray soliton away from its original trajectory (indicated by the red dashed line in Fig. 1(d)), and then enable it transformed into a black soliton with a quasi-static state. The output soliton profile in Fig. 1(b) also shows that careful power adjustment of the probe wave can enable the gray soliton with slower velocity (relative to the group-velocity at the carrier frequency) converted into a black soliton through the nonlinear interaction. Therefore, we can conclude that the optical event horizon can directly induce the reversible conversion between a black soliton and a gray one.

To better understand the above conversion process for dark solitons at optical event horizon, we derive the expression of probeinduced nonlinear phase shift based on the coupled nonlinear Schrödinger equations (CNLSEs) [21, 31]. The induced nonlinear phase shift of a dark soliton from cross-phase modulation (XPM) at the propagation distance $Z=L_{c}$ is well approximated by [32-34]:

$$
\varphi_{X P M}=2 R \cdot\left(D_{m i s}^{\prime} / L_{c}\right) \cdot P_{p} \cdot\left|A_{p}\left(L_{c}, T\right)\right|^{2},
$$

where

$$
R=\frac{\cosh ^{2}\left(\frac{\pi}{2} \sqrt{\frac{16}{\left|\beta_{2}\left(\Omega_{G V M}\right)\right|}-1}\right)}{\cosh ^{2}\left(\frac{\pi}{2} \sqrt{\frac{16}{\left|\beta_{2}\left(\Omega_{G V M}\right)\right|}-1}\right)+\sinh ^{2}\left(\frac{\pi \Delta \Omega}{\cos \phi}\right)}
$$

is the reflection coefficient of the probe wave on the dark soliton associated with the grayness parameter, $\Delta \Omega$ is the frequency shift from $\Omega_{G V M}=\left(-1-\sqrt{1+2 \sin \phi \cdot \delta_{3}}\right) / \delta_{3}$, and $D_{m i s}^{\prime}$ is the velocity mismatch between the dark soliton and the probe wave. From equation (6) we can expect that the reflected part of the probe wave in terms of the intensity, time delay and relative carrier frequency allows an accurate control of the nonlinear phase shift imposed on the dark solitons. However, among all these parameters, the peak power of the probe wave can be simply adjusted and considered as the most effective approach to tuning the XPM-induced nonlinear phase shift of dark soliton at optical event horizon.

The output profiles of dark solitons under the action of the probe wave with three certain different power levels are calculated and displayed in Fig. 2. The relatively weak probe wave $\left(\mathrm{P}_{\mathrm{p}}=0.016\right)$ can enable the gray soliton transformed into one with larger grayness (shown in red curve in the Fig. 2(a)). This is attributed to the fact that the XPM-induced nonlinear phase shift of the dark soliton can counteract a part of the internal phase of the gray soliton itself when the probe wave collides with the dark soliton. When the peak power of the probe wave increases to, $P_{p}=0.026$, which can provide a nonlinear phase shift that exactly balances that of the gray soliton itself, the input gray soliton is transformed into a black one (the green curve). However, further increasing the peak power of the probe wave $\left(\mathrm{P}_{\mathrm{p}}=0.036\right)$ will transform the input soliton into another gray one but with $\phi<0$ (the orange curve) since the probe-induced nonlinear phase shift dominates. The corresponding output simulation results in the input dark soliton with $\phi<0$ are demonstrated in Fig. 2(b). The XPM-induced nonlinear phase shift further enhances the intrinsic phase of the gray soliton, which directly results in the reduced grayness of the transformed dark soliton as the peak power of the probe wave is increasing. This could be intuitively anticipated, since increasing the peak power of the probe wave is equivalent to an increase of the nonlinear phase shift of dark soliton induced by the collision between both pulses at optical event horizon.
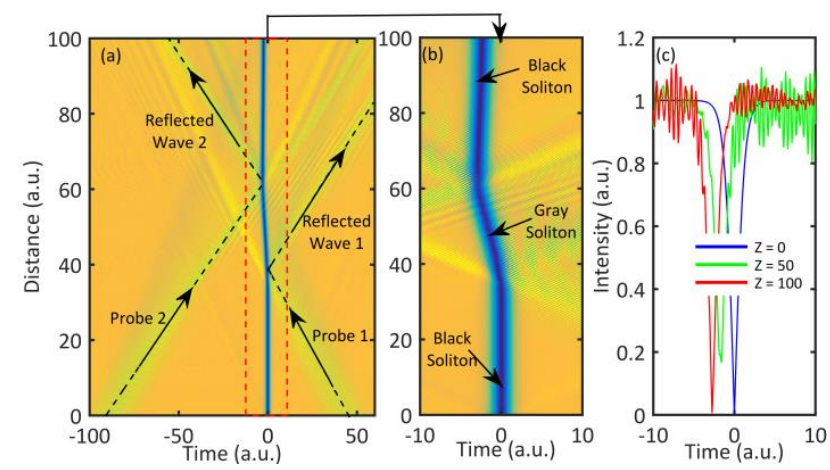

Fig. 3. Dynamics of the dark soliton with Black-Gray-Black transformation as two optical event horizons are cascaded. (a) The temporal evolution during the propagation in the fiber. (b) Zoom in of the temporal evolution of dark soliton (from -10 to 10). (c) The temporal profiles of the dark soliton at different propagation distances.

Considering the time-reversal symmetry for the black- and white-hole horizons $[1,9,18]$ it is extremely possible to realize the longitudinal dark soliton tunneling, where the dark soliton transformation at optical event horizon can be reset purposely. To verify this, we consider a cascade of two optical event horizon analogues. The initial condition in this case is changed to

$$
\begin{aligned}
A(0, T)= & A_{d s}(0, T)+\sqrt{P_{p}}\left[\exp \left(-\left(T+T_{1}\right)^{2} / w^{2}\right) \exp \left(-i \omega_{1} T\right)\right. \\
& \left.+\exp \left(-\left(T+T_{2}\right)^{2} / w^{2}\right) \exp \left(-i \omega_{2} T\right)\right]
\end{aligned}
$$

where $T_{1}, T_{2}$ corresponds to the launched time delay of the two probe waves relative to the dark soliton, and signs of their values (positive or negative) represents whether the probe wave is ahead or behind of the dark soliton respectively, and $\omega_{1}, \omega_{2}$ denote the relative carrier frequency of the probe wave that satisfies the phasematching condition $[9,12]$. Figure 3 shows the simulated temporal dynamics along the propagation coordinate in the fiber for an input black soliton. The soliton propagation in such a system can be divided into three parts. In the initial stage, the black soliton propagates with almost no-perturbation since no nonlinear interaction with the probe waves is introduced. Subsequently, at certain propagation distance the black soliton collides with the probe wave 1 , and then is converted into a faster gray soliton. Ultimately, the collision between the newly transformed gray soliton and the probe wave 2 will occur once again, where the gray soliton is temporally reshaped into a black one and stays as the quasi-static propagation till the output port. This process is analogous to the quantum tunneling effect.

The same design we have employed for an input black soliton can also be applied to the control and recovery of gray soliton. We plot one such example (for the slower gray soliton) in Fig. 4. We observe the transformation of the light pulse from a gray soliton into a purely black one and then back into a gray one with nearly unchanged parameters (with respect to their initial values). These results provide numerical evidence for the longitudinal dark soliton tunneling process as the cascaded system consisting of two optical event horizons is applied. Although several parallel mechanisms $[35,36]$ that are responsible for the soliton tunneling effect have been given, the transformation and control of dark solitons 
concerning a purely black or gray soliton recovery is demonstrated for the first time, to the best of our knowledge, in our system through careful adjustment of the peak power of an additional weak probe wave. In both examples discussed here, the intrinsic manipulation mechanism is believed to a wave blocking phenomenon that is also known from hydrodynamical systems.

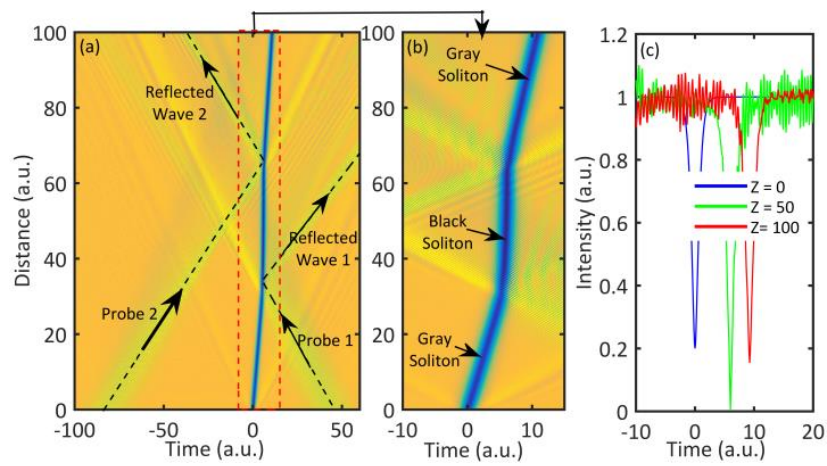

Fig. 4. Dynamics of the dark soliton with Gray-Black-Gray transformation as two optical event horizons are cascaded. The other is the same as Fig. 3.

In conclusion, we have demonstrated that the temporal reflection of an optical pulse at optical event horizon provides an unprecedented potential to control the properties of a dark soliton through another weak probe pulse. By increasing the probe power with respect to the soliton, one can easily modify the dark soliton properties through XPM, opening the way to all-optical signal processing applications. When the cascaded system consisting of two optical event horizons is applied, we observed a new optical soliton tunneling effect where a dark soliton can longitudinally be reconstructed without experiencing substantial variations of its properties. Longitudinal dark soliton tunneling is a clear indication for a high degree of reproducibility of the temporal pulse, and provides a fundamentally new example of optical invisibility, which may find applications in the cloaking of information.

Funding. The European Horizon 2020-MSCA-IF (701493); National Natural Science Fund Foundation of China (NSFC) (61875132, 61505124, 61505122, 61605166); Shenzhen Government's Plan of Science and Technology (JCYJ20170302153731930); Natural Science Foundation of SZU (2017022).

\section{References}

1. T. G. Philbin, C. Kuklewicz, S. Robertson, S. Hill, F. König, and U. Leonhardt, Science, 319(5868), 1367-1370 (2008).

2. K. E. Webb, M. Erkintalo, Y. Xu, N. G. Broderick, J. M. Dudley, G. Genty, and S. G. Murdoch, Nat. Commun. 5:4969, 1-7 (2014).

3. A. V. Yulin, D. V. Skryabin, and P. S. J. Russell, Opt. Lett., 29(20), 2411-2413 (2004)

4. A. Efimov, A.V. Yulin, D.V. Skryabin, J.C. Knight, N. Joly, F.G. Omenetto, A.J. Taylor, and P. Russell, Phys. Rev. Lett. 95(21), 213902 (2005).

5. A. Demircan, S. Amiranashvili, and G. Steinmeyer, Phys. Rev. Lett. 106(16), 163901 (2011).

6. P. Kanakis, and T. Kamalakis, Opt. Lett. 41(7), 1372-1375 (2016).

7. Z. X. Deng, J. Liu, X. W. Huang, C. J. Zhao, and X. L. Wang, Opt. Express 25(23), 28556-28566 (2017).
8. V. Mishra, R. Haldar, P. Mondal, and S. K. Varshney, J. Lightwave Techn. 36(13), 2582-2588 (2018).

9. Z. X. Deng, X. H. Shi, C. Tan, and X. Q. Fu, J. Opt. Soc. Am. B 33(5), 857-863 (2016).

10. A. V. Yulin, R. Driben, B. A. Malomed, and D. V. Skryabin, Opt. Express 21(12), 14481-14486 (2013).

11. Z. X. Deng, X. Q. Fu, J. Liu, C. J. Zhao, and S. C. Wen, Opt. Express 24(10), 10302-10312 (2016).

12. I. Oreshnikov, R. Driben, and A. V. Yulin, Opt. Lett. 40(21), 4871-4874 (2015).

13. T. Marest, C. M. Arabi, M.Conforti, A. Mussot, C. Milian, D. V. Skryabin, and A. Kudlinski, Opt. Express 26(18), 23480-23491 (2018).

14. A. Demircan, S. Amiranashvili, C. Bree, C. Mahnke, F. Milschke, and G. Steinmeyer, Sci. Rep. 2: 850 (2012).

15. I. Oreshnikov, R. Driben, and A. V. Yulin, Opt. Lett. 40(23), 5554-5557 (2015).

16. S. F. Wang, A. Mussot, M. Conforti, A. Bendahmane, X. L. Zeng, and A. Kudlinski, Phys. Rev. A 92(2), 023837 (2015).

17. S. F. Wang, A. Mussot, M. Conforti, X. L. Zeng, and A. Kudlinski, Opt. Lett. 40(14), 3320-3323 (2015).

18. Z. X. Deng, J. Liu, X. W. Huang, C. J. Zhao, and X. L. Wang, Opt. Express 26(13), 16535-16546 (2018).

19. Y. S. Kivshar, and B. Luther-Davies, Phys. Rep. 298(2/3), 81-197 (1998).

20. M. Nakazawa, and K. Suzuki, Electron. Lett. 31(4), 1076-1084 (1995).

21. G. P. Agrawal, Nonlinear Fiber Optics, $4^{\text {th }}$ ed. (Academic, 2007).

22. A. Maruta, and Y. Kodama, Opt. Lett. 20(17), 1752-1754 (1995).

23. H. Ikeda, M. Matsumoto, and A. Hasegawa, Opt. Lett. 20(10), 1113-1115 (1995).

24. S. Robertson, and U. Leonhardt, Phys. Rev. A 81(6), 063835 (2010).

25. V. V. Afanasjev, C. R. Menyuk, and Y. S. Kivshar, Opt. Lett. 21(24), 19751977 (1996)

26. A. Mahalingam, and K. Porsezian, Phys. Rev. E, 64(4), 046608 (2001).

27. C. Milián, D. V. Skryabin, and A. Ferrando, Opt. Lett. 34(14), 2096-2098 (2009).

28. T. Marest, C. M. Arabí, M. Conforti, A. Mussot, C. Milián, D. V. Skryabin, and A.Kudlinski, Opt. Lett. 41(11), 2454-2457 (2016).

29. T. Marest, C. M. Arabí, M. Conforti, A. Mussot, C. Milián, D. V. Skryabin, and A. Kudlinski, Opt. Lett. 43(7), 1511-1514 (2018).

30. D. Krökel, N. J. Halas, G. Giuliani, and D. Grischkowsky, Phys. Rev. Lett., 60(1), 29 (1988).

31. S. Pickartz, U. Bandelow, and S. Amiranashvili, Phys. Rev. A 94(3), 033811 (2016).

32. J. Gu, H. Guo, S. Wang, and X. Zeng, Opt. Express 23(17), 22285-22290 (2015)

33. A. Choudhary, and F. König. Opt. Express 20(5), 5538-5546 (2012).

34. C. M. Arabí, F. Bessin, A. Kudlinski , A. Mussot, D. V. Skryabin, and M. Conforti, Phys. Rev. A 94(6), 063847 (2016).

35. P. Sprenger, M. A. Hoefer, and G. A. El, Phys. Rev. E, 97(3), 032218(2018).

36. T. Marest, F. Braud, M. Conforti, S. Wabnitz, A. Mussot, and A. Kudlinski, Opt. Lett., 42(12), 2350-2353 (2017). 
INFORMATIONAL FIFTH PAGE

\section{REFERENCES}

1. T. G. Philbin, C. Kuklewicz, S. Robertson, S. Hill, F. König, and U. Leonhardt, "Fiber-optical analog of the event horizon," Science, 319(5868), 13671370 (2008)

2. K. E. Webb, M. Erkintalo, Y. Xu, N. G. Broderick, J. M. Dudley, G. Genty, and S. G. Murdoch, "Nonlinear optics of fibre event horizons," Nat. Commun. 5:4969, 1-7 (2014).

3. A. V. Yulin, D. V. Skryabin, and P. S. J. Russell, "Four-wave mixing of linear waves and solitons in fibers with higher-order dispersion", Opt. Lett., 29(20), 2411-2413 (2004).

4. A. Efimov, A.V. Yulin, D.V. Skryabin, J.C. Knight, N. Joly, F.G. Omenetto, A.J. Taylor, and P. Russell, "Interaction of an Optical Soliton with a Dispersive Wave", Phys. Rev. Lett. 95(21), 213902 (2005).

5. A. Demircan, S. Amiranashvili, and G. Steinmeyer, "Controlling light by light with an optical event horizon," Phys. Rev. Lett. 106(16), 163901 (2011).

6. P. Kanakis and T. Kamalakis, "Enabling transistor-like action in photonic crystal waveguides using optical event horizons," Opt. Lett. 41(7), 1372 1375 (2016)

7. Z. X. Deng, J. Liu, X. W. Huang, C. J. Zhao, and X. L. Wang, "Active control of adiabatic soliton fission by external dispersive wave at optical event horizon," Opt. Express 25(23), 28556-28566 (2017).

8. V. Mishra, R. Haldar, P. Mondal, and S. K. Varshney, "Efficient all-Optical transistor action in short-length multimode optical fibers," J. Lightwave Techn. 36(13), 2582-2588 (2018).

9. Z. X. Deng, X. H. Shi, C. Tan, and X. Q. Fu, "Reversible conversion between optical frequencies of probe and idler waves in regime of optical event horizon," J. Opt. Soc. Am. B 33(5), 857-863 (2016).

10. A. V. Yulin, R. Driben, B. A. Malomed, and D. V. Skryabin, "Soliton interaction mediated by cascaded four wave mixing with dispersive waves," Opt. Express 21(12), 14481-14486 (2013).

11. Z. X. Deng, X. Q. Fu, J. Liu, C. J. Zhao, and S. C. Wen, "Trapping and controlling the dispersive wave within a solitonic well," Opt. Express 24(10), 10302-10312 (2016).

12. I. Oreshnikov, R. Driben, and A. V. Yulin, "Weak and strong interactions between dark solitons and dispersive waves," Opt. Lett. 40(21), 48714874 (2015).

13. T. Marest, C. M. Arabi, M.Conforti, A. Mussot, C. Milian, D. V. Skryabin, and A. Kudlinski, "Collision between a dark soliton and a linear wave in an optical fiber," Opt. Express 26(18), 23480-23491 (2018).

14. A. Demircan, S. Amiranashvili, C. Bree, C. Mahnke, F. Milschke, and G. Steinmeyer, "Rogue events in the group velocity horizon," Sci. Rep. 2: 850 (2012).

15. I. Oreshnikov, R. Driben, and A. V. Yulin, "Interaction of high-order solitons with external dispersive waves," Opt. Lett. 40(23), 5554-5557 (2015).

16. S. F. Wang, A. Mussot, M. Conforti, A. Bendahmane, X. L. Zeng, and A. Kudlinski, "Optical event horizons from the collision of a soliton and its own dispersive wave," Phys. Rev. A 92(2), 023837 (2015).

17. S. F. Wang, A. Mussot, M. Conforti, X. L. Zeng, and A. Kudlinski, "Bouncing of a dispersive wave in a solitonic cage," Opt. Lett. 40(14), 3320-3323 (2015).

18. Z. X. Deng, J. Liu, X. W. Huang, C. J. Zhao, and X. L. Wang, "Dark solitons manipulation using optical event horizon," Opt. Express 26(13), 1653516546 (2018).

19. Y. S. Kivshar, and B. Luther-Davies, "Dark optical solitons: physics and applications," Phys. Rep. 298(2/3), 81-197 (1998).

20. M. Nakazawa, and K. Suzuki, Electron. Lett. 31(4), 1076-1084 (1995).

21. G. P. Agrawal, Nonlinear Fiber Optics, $4^{\text {th }}$ ed. (Academic, 2007).

22. A. Maruta, and Y. Kodama, "Suppression of interactions between adjacent optical dark solitons by mean of synchronized phase modulation," Opt. Lett. 20(17), 1752-1754 (1995).

23. H. Ikeda, M. Matsumoto, and A. Hasegawa, "Transmission control of dark solitons by means of nonlinear gain," Opt. Lett. 20(10), 1113-1115 (1995).
24. S. Robertson and U. Leonhardt, "Frequency shifting at fiber-optical event horizons: the effect of Raman deceleration," Phys. Rev. A 81(6), 063835 (2010).

25. V. V. Afanasjev, C. R. Menyuk, and Y. S. Kivshar, "Effect of third-order dispersion on dark solitons," Opt. Lett. 21(24), 1975-1977 (1996).

26. A. Mahalingam, and K. Porsezian, "Propagation of dark solitons with higher-order effects in optical fibers," Phys. Rev. E, 64(4), 046608 (2001).

27. C. Milián, D. V. Skryabin, and A. Ferrando, "Continuum generation by dark solitons," Opt. Lett. 34(14), 2096-2098 (2009).

28. T. Marest, C. M. Arabí, M. Conforti, A. Mussot, C. Milián, D. V. Skryabin, and A.Kudlinski, "Emission of dispersive waves from a train of dark solitons in optical fibers," Opt. Lett. 41(11), 2454-2457 (2016).

29. T. Marest, C. M. Arabí, M. Conforti, A. Mussot, C. Milián, D. V. Skryabin, and A. Kudlinski, "Grayness-dependent emission of dispersive waves from dark solitons in optical fibers," Opt. Lett. 43(7), 1511-1514 (2018).

30. D. Krökel, N. J. Halas, G. Giuliani, and D. Grischkowsky, "Dark-pulse propagation in optical fibers", Phys. Rev. Lett., 60(1), 29 (1988).

31. S. Pickartz, U. Bandelow, and S. Amiranashvili, "Adiabatic theory of solitons fed by dispersive waves," Phys. Rev. A 94(3), 033811 (2016).

32. J. Gu, H. Guo, S. Wang, and X. Zeng, "Probe-controlled soliton frequency shift in the regime of optical event horizon," Opt. Express 23(17), 2228522290 (2015).

33. A. Choudhary, and F. König. "Efficient frequency shifting of dispersive waves at solitons," Opt. Express 20(5), 5538-5546 (2012).

34. C. M. Arabí, F. Bessin, A. Kudlinski , A. Mussot, D. V. Skryabin, and M. Conforti, "Efficiency of four-wave mixing between orthogonally polarized linear waves and solitons in a birefringent fiber," Phys. Rev. A 94(6), 063847 (2016).

35. P. Sprenger, M. A. Hoefer, and G. A. El, “Hydrodynamic optical soliton tunneling", Phys. Rev. E, 97(3), 032218(2018).

36. T. Marest, F. Braud, M. Conforti, S. Wabnitz, A. Mussot, and A. Kudlinski, "Longitudinal soliton tunneling in optical fiber", Opt. Lett., 42(12), 23502353 (2017). 